How to cite this article:

Sukendar, I., Sugiyono, A., \& Munfiqotusshifa. (2020). Medicine Invetory Control By Considering Expiry Periods And Product Returns Using The Always Better Control (ABC) Analysis And The Handley Within Model Of Economic Order Quality (EOQ) At Pharmacies In Indonesia. Journal of Technology and Operations Management, 15(2), $20-32$. https://doi.org/10.32890/jtom2020.15.2.3

\title{
MEDICINE INVENTORY CONTROL BY CONSIDERING EXPIRY PERIODS AND PRODUCT RETURNS USING THE ALWAYS BETTER CONTROL (ABC) ANALYSIS AND THE HANDLEY WITHIN MODEL OF ECONOMIC ORDER QUALITY (EOQ) AT PHARMACIES IN INDONESIA
}

\author{
${ }^{1}$ Irwan Sukendar, ${ }^{2}$ Andre Sugiyono, \& ${ }^{3}$ Munfiqotusshifa \\ Department of Industrial Engineering Faculty of Industrial Technology, Universitas Islam Sultan Agung \\ Semarang Indonesia Jl. Raya Kaligawe KM.4 Semarang Indonesia \\ Corresponding author: irwansukendar@gmail.com
}

Received: 24/7/2020 Revised: 26/8/2020 Accepted: 24/11/2020 Published: 29/12/2020

\begin{abstract}
This study aims to control medicine inventories by considering the expiration period and the product return using The Always Better Control (ABC) analysis and The handley within model of Economic Order Quantity (EOQ). The results of this study indicate that there are $21 \%$ of medicines or 22 types of medicines belonging to group A with the use of $74.64 \%$. for group B there were $25 \%$ drugs or 28 types of drugs with a budget use of $15.31 \%$ of all medicines. Meanwhile, there are $55 \%$ of medicines or 60 types of medicines belonging to group $\mathrm{C}$ with the use of a budget of $10.05 \%$ of the total medicines and the calculation using the EOQ method by considering the expiration period and product returns in this research has a more optimal order size compared to the previous method so that it can minimize expired medicines and estimate which medicines will expire at the end of the cycle so that the total cost of supplies at the pharmacy is more optimal.
\end{abstract}

Keywords: Inventory control, $\mathrm{ABC}$ analysis, EOQ, expiration.

\section{INTRODUCTION AND LITERATURE REVIEW}

The development of increasingly modern science and technology has made competition between companies tighter, along with the many new companies that have sprung up in the same industry. The pharmaceutical industry is one of the industries that continues to grow with intense competition, namely the pharmacy. the tight competition of pharmacies so that good operational management is 
needed to keep the business running. Good management by carrying out an efficient, effective, and economical inventory planning. Pharmacy is included in the category of trading companies because its main activity is to purchase drug supplies from drug distributors or suppliers to be resold to consumers without changing the form or function of the goods. So that the role of supply is an important component in pharmacy operational activities (Rachmawati, Syafirullah, \& Faiz, 2020). According to (Apriyani \& Muhsin, 2017) Inventory control is an activity in managing inventory to suit your needs and remain stable.

And currently drug checks are carried out every day by the pharmacy and it is known that the demand for generic drugs in pharmacies is quite high, but it is not balanced with good inventory control so that inventory control is not optimal. To place an order, the pharmacy only looks at previous medicine consumption and orders are made when the medicine has reached a supply crisis limit. And because medicine is a perishable product category where the value of the product will decrease over time or cannot be used again if it has passed its expiration date. So if there is a medicine that has passed the expiration limit or damage will cause high inventory costs. And medicines that are not in demand until a certain time limit will be returned with several conditions from the distributor. If the requirements for return are not met, the medicine cannot be returned so that the medcine will be destroyed with the cost of destruction being borne by the pharmacy which results in losses for the pharmacy. The above problems can be avoided if the pharmacy manages to control medicine supplies properly. So far, pharmacies have not carried out inventory control calculations by considering product expiration and returns. to find out the types of medicines that require close supervision in terms of inventory, the size of the order that should be made and the right time to reorder so as to minimize the total cost of supplies.

Several previous studies on medicines supply control are (Nafisah, Puryani, \& Lukito, 2011) where in that study developed an EOQ model for pharmaceutical products by considering the expiration period and product returns. And research (Buwono, Priyandari, \& Jauhari, 2014), (Hermanto, Indrajaya, \& Suhendar, 2018) which only developed the EOQ method in their research so that it was developed again by (Resmana \& Rukmayadi, 2019), (Ulfa, Said Salim Dahda, \& Widyaningrum, 2018). For research (Dyatmika \& Krisnadewara, 2017) by adding the ABC method to classify goods based on investment. From the limitations of previous studies, researchers finally developed it again (Alfanda, Pujotomo, \& Wp, 2018) using the EOQ and ABC methods by considering the expiration period and product returns.

\section{RESEARCH OBJECTIVES}

The purpose of this study is to classify medicinal products based on the level of importance and size of investment, determine the optimal order size and estimate the medicines that will expire at the end of the cycle so as to reduce the total cost of supplies by using the $\mathrm{ABC}$ analysis and Economy Order Quantity (EOQ).

\section{RESEARCH METHODOLOGY AND DATA ANALYSIS}

This research begins with a preliminary study, namely a field study, conducting interviews with the person in charge and employees at the pharmacy to obtain information and data needed in the study. Then identify the problem and determine the purpose of the research, then collect data that will be used in the research, then proceed with processing the data that has been developed and then carry out analysis, finding and interpretation.

\section{Model Formulation}

Based on previous research (Alfanda et al., 2018), researchers will perform data processing with different case studies, where in this study a probabilistic EOQ model for pharmaceutical products was 
developed by considering the expiration period and product returns so as to prove that the method used can minimize inventory costs. The assumptions used in this study are as follows:

1. Demand is probabilistic because disease cannot be predicted

2. Constant order size for each order, ordering is only made when inventory reaches reorder point (r).

3. Constant price of goods $(\mathrm{P})$ both to the quantity of goods ordered and time.

4. Shortage of inventory is calculated by backorder.

5. Order charge (A) is constant for each order regardless of the quantity ordered.

6. The cost of holding is proportional to the amount of inventory.

7. The expiration period is known.

8. Drugs that have expired cannot be resold.

9. Medicines that can be returned to suppliers before the expiration date

10. The medcines returned will be replaced with the same drug with a longer expiration period.

11. If the medicine is less than one lot, the drug cannot be returned to the supplier.

12. Medicines that have expired will be worth Rp. 0 .,

Notations :

$Q^{*} \quad$ : optimal lot (item)

$A \quad$ : request cost (Rp/pesan)

$D \quad$ : Demand (item)

$H \quad$ : holding cost $(\mathrm{Rp})$

$\mathrm{Cu} \quad$ : stockout $\operatorname{cost}(\mathrm{Rp})$

$E R^{*}$ : estimated number of products that will expire (item)

$P \quad$ : unit price $(\mathrm{Rp})$

$A R \quad:$ return cost $(\mathrm{Rp})$

$r \quad:$ reorder point (item)

L : leadtime (year)

$w \quad$ : returned product number (box)

$s \quad$ : units per product that can be returned (item)

$n \quad:$ the number of product returns in one period

a : the possibility of an inventory shortage

$\mathrm{Za}$ : value the possibility of inventory shortages

$\mathrm{S} \quad$ : standar deviasi of demand

$N \quad$ : expected number of items deficient

$m \quad$ : fixed expiration period

$D L \quad$ : demand for leadtime

$E A C$ : inventory cost per cycle $(\mathrm{Rp})$

\section{Grouping Medicine Based on ABC Analysis}

This method is an analysis that is used solely to sort the number of uses, then classify the types of goods in an effort to find out the types of goods in an effort to find out the types of medcines movement which include various types, lots of quantities and different patterns of need (Assauri, 2004). The steps in processing medcines grouping data based on $\mathrm{ABC}$ analysis are as follows: The steps are :

1. Determine the number of items for each type of item

2. Determine the price per item for each type of item

3. Multiplying the price per item by the number of units to determine the total investment value of each type of item

4. Arrange the order of the types of goods according to the amount of the total investment value, where the largest total investment value is in the first order.

5. Calculate the cumulative percentage of goods from the many types of goods

6. Calculate the cumulative percentage of the investment value of goods from the total investment value.

7. Grouping classes based on the percentage of goods and the percentage of the investment value of goods. 
8. Draw an $\mathrm{ABC}$ analysis curve or a pareto chart to show the level of importance

\section{Handley Within Economy Order Quantity model}

Economic Order Quantity (EOQ) is a number of inventory items that can be ordered during a period for the purpose of minimizing the cost of these goods (Sabarguna, 2004). According to (Sukanta, 2017) to determine the value of q) * and $r$, it is searched using Handley Within model with lost sales using iteration. The first step that must be taken is to calculate the q value with the Wilson formula, as follows:

$$
\begin{aligned}
& q_{01}^{*}=\sqrt{\frac{2 A D}{h}} \\
& a=\frac{h q_{01}^{*}}{C_{u} D+h q_{01}^{*}} \\
& Z \propto=1-\propto \\
& r_{1}^{*}=D L+Z \alpha S \sqrt{L} \\
& q_{02}^{*}=\sqrt{\frac{2 D\left(A+C_{u} N\right.}{h}} \\
& \int_{r}^{\infty}(x-r) f(x) d x=S_{L}[f(Z \alpha)-Z \propto \psi(Z \alpha)]
\end{aligned}
$$

The values for $\mathrm{f}(\mathrm{Za})$ and $\psi(\mathrm{Z} \alpha)$ are obtained from the Standard Normal Deviation table. Then the value of $\mathrm{q}_{2}^{*}$ is obtained, so the value of $\mathrm{r}_{2}^{*}$ can be determined with the same steps. After the $\mathrm{r}_{1}^{*}$ and $\mathrm{r}_{2}{ }_{2}$ values are obtained, the two values can be compared, if the two values do not have a significant difference and are almost the same then the shrimp paste is finished. Conversely, if the value is significantly different, the processing is continued with the next iteration with the same rate. $r$ here is denoted by the reorder point. Where the reorder point according to (Rangkutty, 2017) is the limit or point of the number of reordering including the demand desired or needed during the grace period follows:

\section{Expected Amount of Expired Products at the end of the cycle (ER)}

$$
E R=\frac{h Q}{\left(h+C_{u}\right)}
$$

\section{Number of Product Returns}

Product returns can be made if there are still s products, where $s$ is the quantity of the product per lot. $\mathrm{w}$ is the number of lots that can be returned and $\mathrm{w}$ is a positive integer. Since $\mathrm{w}$ is the unit of return, only the ER that can be calculated is as follows:

$$
\begin{aligned}
& W=\frac{E R}{S} \\
& O_{E}=\frac{P(E R-W S)^{2}}{2 Q}
\end{aligned}
$$




$$
O_{R}=A_{R}
$$

\section{Total Inventory Cost}

Total Inventory cost $=P D+\frac{h(Q-E R)}{2 Q}+\frac{A D}{Q}+\frac{C_{u}(E R)^{2}}{2 Q}+\frac{P(E R-w \cdot s)^{2}}{2 Q}+A_{R}$

\section{Results}

Tabel 1

Medicines Grouping Based on ABC Analysis

\begin{tabular}{lcccc}
\hline $\begin{array}{c}\text { Medicines } \\
\text { group }\end{array}$ & $\begin{array}{c}\text { Group } \\
\text { number }\end{array}$ & Percentages & value (Rp) & percentages \\
\hline Group A & 22 & $20 \%$ & Rp. 403.103 .376 & $75 \%$ \\
Group B & 28 & $25 \%$ & Rp. 82.701 .635 & $15 \%$ \\
Group C & 60 & $55 \%$ & Rp. 54.278 .684 & $10 \%$ \\
Total & 110 & $100 \%$ & Rp. 540.083.695 & $100 \%$ \\
\hline
\end{tabular}

Furthermore, the EOQ analysis was carried out. The data used in the EOQ calculation are ordering costs which include telephone costs, quota costs, paper costs. So that the total of each message in this study is Rp. 10,860. For a saving cost of $20 \%$ of the purchase price for one drug item. Where the saving cost is the cost that arises when the company stores the product in a warehouse storage area (Vrat, 2014).

Tabel 2

Recapitulation of Medicines Supply Planning

\begin{tabular}{|c|c|c|c|c|c|c|c|c|c|}
\hline No. & Medicines & Units & $\mathbf{Q}$ & ROP & SS & interval & ER & $\mathbf{s}$ & $\mathbf{w}$ \\
\hline 1 & Herbesser Cd 200 & Tablet & 79 & 13,89 & 7 & 24 & 13 & 30 & 0 \\
\hline 2 & $\mathrm{Hb}$ Vit Kaplet 30s & Tablet & 59 & 10,60 & 5 & 22 & 10 & 100 & 0 \\
\hline 3 & Valsartan Tab 80mg & Tablet & 392 & 64,25 & 23 & 48 & 65 & 30 & 2 \\
\hline 4 & Promag Tab Str $12 \mathrm{~s}$ & Tablet & 216 & 27,84 & 15 & 33 & 36 & 30 & 1 \\
\hline 5 & Neurofenac Plus Tab 100s & Tablet & 230 & 26,87 & 11 & 29 & 38 & 100 & 0 \\
\hline 6 & Glimepirid Tab $2 \mathrm{Mg}$ & Tablet & 319 & 33,95 & 15 & 34 & 53 & 50 & 1 \\
\hline 7 & Vitamin B Complex & Tablet & 295 & 45,67 & 15 & 39 & 49 & 100 & 0 \\
\hline 8 & Fionat $\mathrm{Tab} 0,7 \mathrm{Mg}$ & Tablet & 269 & 47,67 & 21 & 50 & 45 & 100 & 0 \\
\hline 9 & $\begin{array}{l}\text { Odansetron Tab } 4 \mathrm{Mg} \\
\text { (E-Cat) }\end{array}$ & Tablet & 60 & 8,80 & 5 & 31 & 10 & 5 & 2 \\
\hline 10 & Amlodipin Tab $10 \mathrm{Mg}$ & Tablet & 1402 & 149,27 & 42 & 65 & 234 & 30 & 8 \\
\hline 11 & $\begin{array}{l}\text { Nitrokaf Retard Kap } \\
\text { Sr 2,5 Mg }\end{array}$ & capsule & 351 & 22,24 & 12 & 36 & 58 & 100 & 0 \\
\hline 12 & $\begin{array}{l}\text { Livron B Plex Tab } \\
\text { Str } 10 \text { s }\end{array}$ & Tablet & 264 & 27,12 & 14 & 39 & 44 & 10 & 5 \\
\hline 13 & $\begin{array}{l}\text { Adalat Oros Tab } 30 \mathrm{Mg} \\
\text { (Nifedipin) }\end{array}$ & Tablet & 132 & 27,43 & 11 & 41 & 22 & 30 & 0 \\
\hline 14 & Digest $30 \mathrm{Mg}$ Cap 20s & Tablet & 253 & 34,98 & 19 & 47 & 42 & 20 & 2 \\
\hline 15 & $\begin{array}{l}\text { Cetirizine Hexpharm } \\
\text { 10mg Tab 50s }\end{array}$ & Tablet & 296 & 26,70 & 12 & 39 & 49 & 50 & 1 \\
\hline 16 & Vometa Flash 10 & Tablet & 196 & 19,08 & 9 & 38 & 33 & 50 & 0 \\
\hline
\end{tabular}




\begin{tabular}{|c|c|c|c|c|c|c|c|c|c|}
\hline & $\mathrm{Mg} \mathrm{Tab}$ & & & & & & & & \\
\hline 17 & $\begin{array}{l}\text { Co Amoxiclave } 625 \\
\mathrm{Mg} \mathrm{Tab}\end{array}$ & Tablet & 147 & 11,17 & 7 & 37 & 25 & 30 & 1 \\
\hline 18 & $\begin{array}{l}\text { Mertigo Sr Tab } \\
100 \mathrm{~S}\end{array}$ & Tablet & 160 & 16,16 & 8 & 38 & 27 & 100 & 0 \\
\hline 19 & Progastic Tab 200s & Tablet & 229 & 20,55 & 10 & 42 & 38 & 200 & 0 \\
\hline 20 & Viaclav $500 \mathrm{Mg}$ & Tablet & 72 & 10,02 & 5 & 39 & 12 & 100 & 0 \\
\hline 21 & $\begin{array}{l}\text { Spironolakton Tab } \\
25 \mathrm{Mg}\end{array}$ & Tablet & 320 & 23,26 & 12 & 59 & 53 & 10 & 6 \\
\hline 22 & Albiotin $300 \mathrm{Mg}$ Tab & Tablet & 269 & 19,55 & 10 & 59 & 45 & 30 & 1 \\
\hline 23 & $\begin{array}{l}\text { Super Tetra } 250 \\
\text { Mg Cap }\end{array}$ & capsule & 595 & 73,38 & 22 & 70 & 99 & 120 & 0 \\
\hline 24 & Herbesser Cd 100 & Tablet & 61 & 6,14 & 4 & 59 & 10 & 30 & 0 \\
\hline 25 & Amlodipin Tab $5 \mathrm{Mg}$ & Tablet & 897 & 103,90 & 47 & 94 & 150 & 30 & 5 \\
\hline 26 & $\begin{array}{l}\text { Azithromycin Tab } \\
500 \mathrm{Mg}\end{array}$ & Tablet & 14 & 2,05 & 2 & 59 & 2 & 20 & 0 \\
\hline 27 & $\begin{array}{l}\text { Miniaspi (Asam } \\
\text { Asetilsalisilat) } 80 \mathrm{Mg}\end{array}$ & Tablet & 1933 & 148,04 & 72 & 101 & 322 & 100 & 3 \\
\hline 28 & Opicef $500 \mathrm{Mg}$ Cap $50 \mathrm{~S}$ & capsule & 46 & 3,13 & 2 & 63 & 8 & 50 & 0 \\
\hline 29 & Clabat F 500 Mg Tab & Tablet & 28 & 5,83 & 4 & 1 & 5 & 20 & 0 \\
\hline 30 & Cefixime Kap $100 \mathrm{Mg}$ & capsule & 189 & 15,49 & 10 & 69 & 31 & 50 & 0 \\
\hline 31 & $\begin{array}{l}\text { Sifrol Er 0,375 Mg } \\
\text { (Pramixsol) }\end{array}$ & Tablet & 18 & 1,30 & 1 & 68 & 3 & 30 & 0 \\
\hline 32 & $\begin{array}{l}\text { Daiticin } 500 \mathrm{Mg} \text { Tab } \\
100 \mathrm{~S}\end{array}$ & Tablet & 718 & 68,60 & 46 & 97 & 120 & 100 & 1 \\
\hline 33 & $\begin{array}{l}\text { Candesartan Dexa } \\
8 \mathrm{mg} \mathrm{Tab}\end{array}$ & Tablet & 89 & 13,01 & 9 & 76 & 15 & 30 & 0 \\
\hline 34 & Binotal 500mg & Tablet & 131 & 20,26 & 15 & 82 & 22 & 50 & 0 \\
\hline 35 & Bioprexium $5 \mathrm{Mg}$ Tab & Tablet & 39 & 5,32 & 3 & 0 & 6 & 30 & 0 \\
\hline 36 & Glimepirid Tab $4 \mathrm{Mg}$ & Tablet & 47 & 9,35 & 6 & 72 & 8 & 50 & 0 \\
\hline 37 & $\begin{array}{l}\text { Acetylcystein } 200 \mathrm{Mg} \\
\text { Capsul }\end{array}$ & capsule & 409 & 90 & 20 & 82 & 68 & 100 & 0 \\
\hline 38 & Primadol Tab & ablet & 355 & 31,90 & 24 & 85 & 59 & 100 & 0 \\
\hline 39 & Arsinal Tab 100s & ablet & 434 & 49,48 & 31 & 4 & 72 & 100 & 0 \\
\hline 40 & Sanprima $480 \mathrm{Mg} \mathrm{Tab}$ & Tablet & 431 & 35,86 & 21 & 84 & 72 & 100 & 0 \\
\hline 41 & Trianta Tab $100 \mathrm{~S}$ & Tablet & 222 & 21,18 & 13 & 82 & 37 & 100 & 0 \\
\hline 42 & Almacon Tab $100 \mathrm{~S}$ & Tablet & 199 & 18,72 & 6 & 79 & 33 & 100 & 0 \\
\hline 43 & Simvastatin $20 \mathrm{Mg}$ Tablet $(\mathrm{Pl})$ & Tablet & 387 & 18,34 & 14 & 81 & 65 & 50 & 1 \\
\hline 44 & Imunos Tab & Tablet & 13 & 0,84 & 1 & 79 & 2.13 & 20 & 0 \\
\hline 45 & $5 \mathrm{mg}$ Tab 30s & Tablet & 125 & 14,65 & 10 & 83 & 21 & 30 & 0 \\
\hline 46 & Bactoprim $9600 \mathrm{Mg}$ Tab $100 \mathrm{~S}$ & Tablet & 695 & 36,24 & 20 & 84 & 116 & 100 & 1 \\
\hline 47 & Lapikot Forte Tab & Tablet & 237 & 23,57 & 15 & 86 & 39 & 100 & 0 \\
\hline 48 & $\begin{array}{l}\text { Ambroxol Bernofarm } \\
30 \mathrm{mg} / 5 \mathrm{ml} \mathrm{Syr}\end{array}$ & Botl & 36 & 6,36 & 5 & 79 & 6 & 100 & 0 \\
\hline 49 & Asam Mefenamat Tab $500 \mathrm{Mg}$ & Tablet & 1071 & 33,37 & 21 & 83 & 178 & 100 & 2 \\
\hline 50 & Betahistin Tab 6mg & Tablet & 382 & 18,80 & 14 & 84 & 64 & 30 & 2 \\
\hline 51 & Lincocin $500 \mathrm{Mg}$ Cap $30 \mathrm{~S}$ & capsule & 35 & 2,29 & 2 & 81 & 6 & 30 & 0 \\
\hline 52 & Vitamin B1 $50 \mathrm{Mg}$ Tab Kf & Tablet & 230 & 10,93 & 8 & 85 & 38 & 100 & 0 \\
\hline 53 & Acarbose Tab $50 \mathrm{Mg}$ & Tablet & 327 & 30,26 & 23 & 95 & 55 & 100 & 0 \\
\hline 54 & Folavicap $400 \mathrm{Mg}$ Tab $100 \mathrm{~S}$ & Tablet & 613 & 77,01 & 54 & 131 & 102 & 100 & 1 \\
\hline 55 & $\begin{array}{l}\text { Clindamycin Cap } 300 \text { Mg (E- } \\
\text { Kat) }\end{array}$ & capsule & 262 & 32,82 & 19 & 98 & 44 & 50 & 1 \\
\hline 56 & Vitamin C 50mg Tab KF & Tablet & 259 & 10,29 & 8 & 94 & 43 & 100 & 0 \\
\hline 57 & Metronidazol Tab 500 MG & Tablet & 41 & 1,21 & 1 & 93 & 7 & 100 & 0 \\
\hline
\end{tabular}




\begin{tabular}{|c|c|c|c|c|c|c|c|c|c|}
\hline 58 & $\begin{array}{l}\text { Alpentin (Gabapentin) } 100 \mathrm{MG} \\
\text { Kapsul }\end{array}$ & capsule & 90 & 5,10 & 3 & 93 & 15 & 50 & 0 \\
\hline 59 & Amoxicilin Tab 500 MG & Tablet & 738 & 26,78 & 19 & 98 & 123 & 100 & 1 \\
\hline 60 & $\begin{array}{l}\text { Antasida Doen Triman Chew } \\
\text { Tab 100s }\end{array}$ & Tablet & 282 & 16,15 & 10 & 97 & 47 & 100 & 0 \\
\hline 61 & Clopidogrel Paphrostab 75 MG & Tablet & 235 & 8,04 & 3 & 96 & 39 & 30 & 1 \\
\hline 62 & Curcuma Soho Tab & Tablet & 32 & 2,29 & 2 & 98 & 5 & 30 & 0 \\
\hline 63 & Paracetamol If $500 \mathrm{MG} \mathrm{Tab} \mathrm{Str}$ & Tablet & 90 & 13,52 & 9 & 102 & 15 & 50 & 0 \\
\hline 64 & Cefadroxil Kap 500 MG & capsule & 234 & 7,59 & 5 & 102 & 39 & 100 & 0 \\
\hline 65 & Metformin Tab 500 MG & Tablet & 2066 & 163,00 & 74 & 139 & 344 & 100 & 3 \\
\hline 66 & Piralen 10 MG Tab & Tablet & 482 & 13,44 & 9 & 104 & 80 & 10 & 10 \\
\hline 67 & $\begin{array}{l}\text { Harnal Ocas (Tamsulosin Hcl } \\
0.4 \mathrm{mg} \text { ) }\end{array}$ & Tablet & 20 & 0,82 & 1 & 104 & 3 & 30 & 0 \\
\hline 68 & $\begin{array}{l}\text { Domperidone Novell 10MG } \\
\text { Tab }\end{array}$ & Tablet & 674 & 16,92 & 11 & 107 & 112 & 30 & 4 \\
\hline 69 & $\begin{array}{l}\text { Paracetamol Kimia Farma } 500 \\
\text { MG }\end{array}$ & caplet & 89 & 4,14 & 3 & 109 & 15 & 100 & 0 \\
\hline 70 & Ampicillin Kf 500 MG Tab & Tablet & 640 & 53,30 & 20 & 11 & 107 & 100 & 1 \\
\hline 71 & Dra & & 18 & & 6 & & 31 & 100 & 0 \\
\hline 72 & $10 \mathrm{mg} \mathrm{Tab}$ & $\mathrm{Ta}$ & 16 & & 4 & & 28 & 30 & 1 \\
\hline 73 & Allop & Tablet & 1254 & 82 & 19 & 1 & 209 & 100 & 2 \\
\hline 74 & Mg Tab & $\mathrm{Tab}$ & 342 & 30 , & 16 & & 57 & 100 & 0 \\
\hline 75 & $\mathrm{ab} 100 \mathrm{~S}$ & $\mathrm{Ta}$ & 801 & 26 , & 13 & & 134 & 100 & 1 \\
\hline 76 & Dep & $\mathrm{Ta}$ & 26 & 3,8 & 3 & & 4 & 100 & 0 \\
\hline 77 & Tab & Tablet & 127 & 6,8 & 5 & & 21 & 100 & 0 \\
\hline 78 & Rite & capsule & 52 & 4,48 & 4 & 121 & 9 & 30 & 0 \\
\hline 79 & $\begin{array}{l}\text { Isosorbid Dinitrat Tab 5mg } \\
\text { (Isdn) }\end{array}$ & Tablet & 1008 & 22 & 14 & 123 & 168 & 100 & 2 \\
\hline 80 & $\begin{array}{l}\text { Cotrimoxazole Erita } 480 \mathrm{Mg} \\
\text { Tab } 100 \mathrm{~S}\end{array}$ & Tablet & 945 & 62,45 & 28 & 136 & 157 & 100 & 1 \\
\hline 81 & Tab $150 \mathrm{Mg}$ & & 73 & 18,71 & 13 & 1 & 122 & 100 & 1 \\
\hline 82 & Tab Novell & Tablet & 364 & 20,15 & 7 & 135 & 61 & 50 & 1 \\
\hline 83 & $\begin{array}{l}\text { Gabapentin } 300 \text { Mg Kapsul } \\
\text { (Ekat) }\end{array}$ & $\mathrm{c}$ & 386 & 1 & 10 & 137 & 64 & 100 & 0 \\
\hline 84 & Bisoprolol Tab 5mg & & 38 & & 7 & & 64 & 30 & 2 \\
\hline 85 & $0 \mathrm{Mg}$ & Tablet & 161 & 10 & 7 & 14 & 27 & 100 & 0 \\
\hline 86 & Citic & Tablet & 22 & 1,91 & 1 & 149 & 4 & 30 & 0 \\
\hline 87 & $\begin{array}{l}\text { Glibenclamide Indofarma } 5 \mathrm{mg} \\
\mathrm{Tab}\end{array}$ & Tablet & 7 & 11,96 & 7 & 152 & 1 & 0 & 1 \\
\hline 88 & Cetymin Tab & capsule & 47 & 2,24 & 1 & 155 & 8 & 50 & 0 \\
\hline 89 & $\begin{array}{l}\text { Captopril Errita 25mg Tab } \\
100 \mathrm{~s}\end{array}$ & Tablet & 3 & 8,37 & 6 & 170 & 6 & 100 & 0 \\
\hline 90 & Ibuprofen 200mg Tab & Tablet & 790 & 11 & 7 & 11 & 132 & 100 & 1 \\
\hline 91 & Omg Tab & Tablet & 83 & 6 & 5 & & 14 & 30 & 0 \\
\hline 92 & $00 \mathrm{mg} \mathrm{Tab}$ & Tablet & 616 & 28,95 & 13 & 18 & 103 & 100 & 1 \\
\hline 93 & Acyc & Tablet & $21^{\prime}$ & 2,4 & 1 & 18 & 36 & 100 & 0 \\
\hline 94 & Dex & capsule & 169 & 11,82 & 7 & 19 & 28 & 50 & 0 \\
\hline 95 & ab 100s & Tablet & 351 & 16,69 & 8 & 19 & 59 & 100 & 0 \\
\hline 96 & 0mg Cap 50s & Tablet & 299 & 3,06 & 2 & 201 & 50 & 50 & 1 \\
\hline 97 & $\begin{array}{l}\text { Vectrine Cap } 300 \mathrm{Mg} \\
\text { (Erdostein) }\end{array}$ & $\mathrm{c}$ & 29 & 1, & 1 & 202 & 4.90 & 20 & 0 \\
\hline 98 & Furosemid Tab $40 \mathrm{Mg}$ & 1 & 68 & 7 , & 4 & 20 & 114 & 200 & 0 \\
\hline 99 & $a b 100 \mathrm{~s}$ & Tablet & 191 & 1,94 & 1 & 21 & 32 & 100 & 0 \\
\hline 100 & Irbesa & Tablet & 75 & 3,36 & 3 & 219 & 13 & 20 & 0 \\
\hline 101 & Trisela 10mg & Tablet & 213 & 8,03 & 4 & 238 & 36 & 100 & 0 \\
\hline
\end{tabular}




\begin{tabular}{|c|c|c|c|c|c|c|c|c|c|}
\hline 102 & Cetrol Tab & Tablet & 136 & 2,05 & 1 & 248 & 23 & 100 & 0 \\
\hline 103 & Meloxicam Tab $15 \mathrm{Mg}$ & Tablet & 82 & 1,50 & 1 & 254 & 14 & 20 & 0 \\
\hline 104 & Meloxicam Tab 7,5 Mg & Tablet & 109 & 1,48 & & 329 & 18 & 20 & 1 \\
\hline 105 & $\begin{array}{l}\text { Mefenamic Acid Hexpharm } \\
\text { 500mg }\end{array}$ & capsule & 185 & 2,22 & 1 & 338 & 31 & 100 & 0 \\
\hline 106 & $\begin{array}{l}\text { Hydrochlorothiazide Tablet } 25 \\
\mathrm{Mg}\end{array}$ & Tablet & 429 & 2,34 & 1 & 364 & 72 & 100 & 0 \\
\hline 107 & Antalgin Kf 500mg Tab & Tablet & 238 & 1,15 & & 372 & 40 & 100 & 0 \\
\hline 108 & Amlc & Tablet & 66 & 0,26 & & 485 & 11 & 10 & 1 \\
\hline 109 & Propanolol Dexa $10 \mathrm{Mg}$ Tab & Tablet & 435 & 1,71 & 1 & 670 & 73 & 100 & 0 \\
\hline 110 & Salbutamol Tab $4 \mathrm{Mg}$ & Tablet & 201 & 0,31 & 0 & 1113 & 34 & 100 & 0 \\
\hline
\end{tabular}

According to (Heizer, 2010), along with the increase in the quantity of goods ordered, the number of orders per year will decrease but the storage cost will increase because the amount of inventory that must be taken care of is more. And based on the results of the above calculations, it is known that for the example Herbesser CD 200mg the order interval is 0.066 years or 24 days. The optimal order lot for this item is as in equation (1) and an example of calculating the optimal ordering quantity on Herbesser CD 200mg with 1200 requests is 79 items.

Tabel 3

Difference in Total Inventory Cost of research results and actual costs

\begin{tabular}{|c|c|c|c|c|c|}
\hline No. & Medicines & units & $\begin{array}{c}\text { Total Inventory } \\
\text { Cost ABC-EOQ } \\
\text { analysis }\end{array}$ & $\begin{array}{l}\text { Total Inventory } \\
\text { actual Cost }\end{array}$ & differences \\
\hline 1 & Herbesser Cd 200mg & Tablet & Rp. 40.993 .703 & Rp. 41.647.680 & $\begin{array}{l}\text { Rp. } \\
653.977\end{array}$ \\
\hline 2 & $\mathrm{Hb}$ Vit Kaplet 30s & Tablet & Rp. 39.130.084 & Rp. 39.862.942 & $\begin{array}{l}\text { Rp. } \\
732.858\end{array}$ \\
\hline 3 & Valsartan Tab 80mg & Tablet & Rp. 38.949.849 & Rp. 39.219.229 & $\begin{array}{l}\text { Rp. } \\
269.379\end{array}$ \\
\hline 4 & Promag Tab Str 12s & Tablet & Rp. 28.676.736 & Rp. 29.115.082 & $\begin{array}{l}\text { Rp. } \\
438.346\end{array}$ \\
\hline 5 & Neurofenac Plus Tab 100s & Tablet & Rp. 28.451.764 & Rp. 28.904.229 & $\begin{array}{l}\text { Rp. } \\
452.465\end{array}$ \\
\hline 6 & Glimepirid Tab $2 \mathrm{Mg}$ & Tablet & Rp. 23.035.638 & 533 & $\begin{array}{l}\text { Rp. } \\
420.895\end{array}$ \\
\hline 7 & Vitamin B Complex & Tablet & Rp. 21.249.267 & Rp. 21.681.024 & Rp.431.757 \\
\hline 8 & Fionat $\mathrm{Tab} 0,7 \mathrm{Mg}$ & Tablet & Rp. 18.247.934 & Rp. 18.679.592 & $\begin{array}{l}\text { Rp. } \\
431.658\end{array}$ \\
\hline 9 & $\begin{array}{l}\text { Odansetron Tab } 4 \text { Mg (E- } \\
\text { Cat) }\end{array}$ & Tablet & Rp. 17.083.919 & Rp. 17.723 .036 & $\begin{array}{l}\text { Rp. } \\
639.117\end{array}$ \\
\hline 10 & Amlodipin Tab $10 \mathrm{Mg}$ & Tablet & Rp. 15.829.912 & Rp.16.158.175 & $\begin{array}{l}\text { Rp. } \\
328.263\end{array}$ \\
\hline 11 & $\begin{array}{l}\text { Nitrokaf Retard Kap Sr 2,5 } \\
\mathrm{Mg}\end{array}$ & capsule & Rp. 14.309.377 & Rp. 14.768.088 & $\begin{array}{l}\text { Rp. } \\
458.711\end{array}$ \\
\hline 12 & Livron B Plex Tab Str 10s & Tablet & Rp. 14.090.652 & Rp. 14.547521 & $\begin{array}{l}\text { Rp. } \\
456.869\end{array}$ \\
\hline 13 & $\begin{array}{l}\text { Adalat Oros Tab } 30 \mathrm{Mg} \\
\text { (Nifedipin) }\end{array}$ & Tablet & Rp. 14.084.224 & Rp. 14.606.222 & $\begin{array}{l}\text { Rp. } \\
521.998\end{array}$ \\
\hline 14 & Digest $30 \mathrm{Mg}$ Cap 20s & Tablet & Rp. 13.777.952 & Rp. 14.228.552 & $\begin{array}{l}\text { Rp. } \\
450.599\end{array}$ \\
\hline 15 & $\begin{array}{l}\text { Cetirizine } \\
\text { Hexpharm10mgtab 50s }\end{array}$ & Tablet & Rp. 12.510 .501 & Rp. 12.968.136 & $\begin{array}{l}\text { Rp. } \\
457.634\end{array}$ \\
\hline
\end{tabular}




\begin{tabular}{|c|c|c|c|c|c|}
\hline 16 & Vometa Flash $10 \mathrm{Mg}$ Tab & Tablet & Rp. 12.281.235 & Rp. 12.773.065 & $\begin{array}{l}\text { Rp. } \\
491.830\end{array}$ \\
\hline 17 & $\begin{array}{l}\text { Co Amoxiclave } 625 \mathrm{Mg} \\
\mathrm{Tab}\end{array}$ & Tablet & Rp. 11.896 .483 & Rp. 12.398.351 & $\begin{array}{l}\text { Rp. } \\
501.869\end{array}$ \\
\hline 18 & Mertigo Sr Tab $100 \mathrm{~S}$ & Tablet & Rp. 11.754.533 & Rp. 12.260 .928 & $\begin{array}{l}\text { Rp. } \\
506.395\end{array}$ \\
\hline 19 & Progastic Tab 200s & Tablet & Rp. 10.253.401 & Rp. 10.739.236 & $\begin{array}{l}\text { Rp. } \\
485.835\end{array}$ \\
\hline 20 & Viaclav $500 \mathrm{Mg}$ & Tablet & Rp. 10.068.409 & Rp. 10.655.956 & $\begin{array}{l}\text { Rp. } \\
587.547\end{array}$ \\
\hline 21 & Spironolakton Tab $25 \mathrm{Mg}$ & Tablet & Rp. 4.782.316 & Rp. 5.260 .320 & $\begin{array}{l}\text { Rp. } \\
478.004\end{array}$ \\
\hline 22 & Albiotin $300 \mathrm{Mg}$ Tab & Tablet & Rp. 4.727.227 & Rp. 5.210 .160 & $\begin{array}{l}\text { Rp. } \\
482.933\end{array}$ \\
\hline 23 & Super Tetra 250 Mg Cap & capsule & Rp. 4.676.701 & Rp. 5.148.365 & $\begin{array}{l}\text { Rp. } \\
471.664\end{array}$ \\
\hline 24 & Herbesser Cd 100 & Tablet & Rp. 4.341.290 & Rp. 4.922.133 & $\begin{array}{l}\text { Rp. } \\
580.842\end{array}$ \\
\hline 25 & Amlodipin Tab $5 \mathrm{Mg}$ & Tablet & Rp. 4.345.255 & Rp. 4.788.352 & $\begin{array}{l}\text { Rp. } \\
443.097\end{array}$ \\
\hline 26 & Azithromycin Tab 500 Mg & Tablet & Rp. 4.012.935 & Rp. 4.935.505 & $\begin{array}{l}\text { Rp. } \\
922.570\end{array}$ \\
\hline 27 & $\begin{array}{l}\text { Miniaspi (Asam } \\
\text { Asetilsalisilat) Tab } 80 \mathrm{Mg}\end{array}$ & Tablet & Rp. 3.869.678 & Rp. 4.309 .643 & $\begin{array}{l}\text { Rp. } \\
439.965\end{array}$ \\
\hline 28 & Opicef $500 \mathrm{Mg}$ Cap $50 \mathrm{~S}$ & capsule & Rp. 3.784.001 & Rp. 4.393.403 & $\begin{array}{l}\text { Rp. } \\
609.402\end{array}$ \\
\hline 29 & Clabat F 500 Mg Tab & Tablet & Rp. 3.675.654 & Rp. 4.364.401 & $\begin{array}{l}\text { Rp. } \\
688.747\end{array}$ \\
\hline 30 & Cefixime Kap 100 Mg & capsule & Rp. 3.365 .853 & Rp. 3.873 .630 & $\begin{array}{l}\text { Rp. } \\
507.778\end{array}$ \\
\hline 31 & $\begin{array}{l}\text { Sifrol Er 0,375 Mg } \\
\text { (Pramixsol) }\end{array}$ & Tablet & Rp. 3.116.217 & Rp. 3.903 .542 & $\begin{array}{l}\text { Rp. } \\
787.325\end{array}$ \\
\hline 32 & Daiticin $500 \mathrm{Mg}$ Tab $100 \mathrm{~S}$ & Tablet & Rp. 3.087.411 & Rp. 3.550.613 & $\begin{array}{l}\text { Rp. } \\
463.201\end{array}$ \\
\hline 33 & Candesartan Dexa 8mg Tab & Tablet & Rp. 2.887.046 & Rp. 3.429.234 & $\begin{array}{l}\text { Rp. } \\
542.188\end{array}$ \\
\hline 34 & Binotal 500mg & Tablet & Rp. 2.879.015 & Rp. 3.401 .006 & $\begin{array}{l}\text { Rp. } \\
521.991\end{array}$ \\
\hline 35 & $\begin{array}{l}\text { Bioprexium } 5 \mathrm{Mg} \mathrm{Tab} \\
\text { (Perindopril Arginnie) }\end{array}$ & Tablet & Rp. 2.868.166 & Rp. 3.485 .862 & $\begin{array}{l}\text { Rp. } \\
617.696\end{array}$ \\
\hline 36 & Glimepirid Tab $4 \mathrm{Mg}$ & Tablet & Rp. 2.853.974 & Rp. 3.448 .655 & $\begin{array}{l}\text { Rp. } \\
594.680\end{array}$ \\
\hline 37 & $\begin{array}{l}\text { Acetylcystein } 200 \mathrm{Mg} \\
\text { Capsul }\end{array}$ & capsule & Rp. 2.839.903 & Rp. 3.330 .656 & $\begin{array}{l}\text { Rp. } \\
490.753\end{array}$ \\
\hline 38 & Primadol Tab & Tablet & Rp. 2.704.056 & Rp. 3.197.498 & $\begin{array}{l}\text { Rp. } \\
493.441\end{array}$ \\
\hline 39 & Arsinal Tab 100s & Tablet & Rp. 2.,604808 & Rp. 3.093.144 & $\begin{array}{l}\text { Rp. } \\
488.336\end{array}$ \\
\hline 40 & Sanprima 480 Mg Tab & Tablet & Rp. 2.519.999 & Rp. 3.012.623 & $\begin{array}{l}\text { Rp. } \\
492.624\end{array}$ \\
\hline 41 & Trianta Tab $100 \mathrm{~S}$ & Tablet & Rp. 2.484.936 & Rp. 2.990 .340 & $\begin{array}{l}\text { Rp. } \\
505.404\end{array}$ \\
\hline 42 & Almacon Tab $100 \mathrm{~S}$ & Tablet & Rp. 2.471.966 & Rp. 2.980 .704 & $\begin{array}{l}\text { Rp. } \\
508.738\end{array}$ \\
\hline
\end{tabular}




\begin{tabular}{|c|c|c|c|c|c|}
\hline 43 & $\begin{array}{l}\text { Simvastatin } 20 \mathrm{Mg} \text { Tablet } \\
(\mathrm{Pl})\end{array}$ & Tablet & Rp. 2.427.424 & Rp. 2.912 .800 & $\begin{array}{l}\text { Rp. } \\
485.376\end{array}$ \\
\hline 44 & Imunos Tab & Tablet & Rp. 2.347 .342 & Rp. 3.208.616 & $\begin{array}{l}\mathrm{Rp} . \\
861.275\end{array}$ \\
\hline 45 & Flunarizin 5mg Tab 30s & Tablet & Rp. 2.339.542 & Rp. 2.863 .200 & $\begin{array}{l}\text { Rp. } \\
523.658\end{array}$ \\
\hline 46 & $\begin{array}{l}\text { Bactoprim } 9600 \mathrm{Mg} \text { Tab } \\
100 \mathrm{~S}\end{array}$ & Tablet & Rp. 2.341 .956 & Rp. 2.821 .420 & $\begin{array}{l}\text { Rp. } \\
479.464\end{array}$ \\
\hline 47 & Lapikot Forte Tab & Tablet & Rp. 2.325.487 & Rp. 2.829.739 & $\begin{array}{l}\text { Rp. } \\
504.252\end{array}$ \\
\hline 48 & $\begin{array}{l}\text { Ambroxol Bernofarm } \\
\text { 30mg/5ml Syr } 60 \mathrm{Ml}\end{array}$ & Botl & Rp. 2.312.594 & Rp. 2.930.585 & $\begin{array}{l}\text { Rp. } \\
617.991\end{array}$ \\
\hline 49 & $\begin{array}{l}\text { Asam Mefenamat Tab } 500 \\
\mathrm{Mg}\end{array}$ & Tablet & Rp. 2.308.269 & Rp. 2.786.117 & $\begin{array}{l}\text { Rp. } \\
477.848\end{array}$ \\
\hline 50 & Betahistin Tab 6mg & Tablet & Rp. 2.259.466 & Rp. 2.745 .858 & $\begin{array}{l}\text { Rp. } \\
486.393\end{array}$ \\
\hline 51 & Lincocin $500 \mathrm{Mg}$ Cap $30 \mathrm{~S}$ & capsule & Rp. 2.239.055 & Rp. 2.860 .546 & $\begin{array}{l}\text { Rp. } \\
621.491\end{array}$ \\
\hline 52 & Vitamin B1 $50 \mathrm{Mg}$ Tab Kf & Tablet & Rp. 2.119.605 & Rp. 2.626.099 & $\begin{array}{l}\text { Rp. } \\
506.494\end{array}$ \\
\hline 53 & Acarbose Tab $50 \mathrm{Mg}$ & Tablet & Rp. 2.069.193 & Rp. 2.567 .755 & $\begin{array}{l}\text { Rp. } \\
498.562\end{array}$ \\
\hline 54 & $\begin{array}{l}\text { Folavicap 400mcg Tab } 100 \\
\text { S }\end{array}$ & Tablet & Rp. 1.989.384 & Rp. 2.460 .523 & $\begin{array}{l}\text { Rp. } \\
471.138\end{array}$ \\
\hline 55 & $\begin{array}{l}\text { Clindamycin Cap } 300 \mathrm{Mg} \\
\text { (E-Kat) }\end{array}$ & capsule & Rp. 1.926 .858 & Rp. 2.419 .333 & $\begin{array}{l}\text { Rp. } \\
492.475\end{array}$ \\
\hline 56 & Vitamin C 50mg Tab Kf & Tablet & Rp. 1.708.266 & Rp. $2,214.347$ & $\begin{array}{l}\text { Rp. } \\
506.081\end{array}$ \\
\hline 57 & Metronidazol Tab $500 \mathrm{Mg}$ & Tablet & Rp. 1.705 .778 & Rp. 2.294.918 & $\begin{array}{l}\text { Rp. } \\
589.140\end{array}$ \\
\hline 58 & $\begin{array}{l}\text { Alpentin (Gabapentin) } 100 \\
\text { Mg Kapsul }\end{array}$ & Tablet & Rp. 1.701.629 & Rp. 2.237.736 & $\begin{array}{l}\text { Rp. } \\
536.107\end{array}$ \\
\hline 59 & Amoxicilin Tab $500 \mathrm{Mg}$ & Tablet & Rp. 1.650 .511 & Rp. 2.135.295 & $\begin{array}{l}\text { Rp. } \\
484.784\end{array}$ \\
\hline 60 & $\begin{array}{l}\text { Antasida Doen Triman } \\
\text { Chew Tab 100s }\end{array}$ & Tablet & Rp. 1.633.913 & Rp. 2.138.861 & $\begin{array}{l}\text { Rp. } \\
504.948\end{array}$ \\
\hline 61 & $\begin{array}{l}\text { Clopidogrel Paphrostab } 75 \\
\mathrm{Mg}\end{array}$ & Tablet & Rp. 1.628 .680 & Rp. 2.126.040 & $\begin{array}{l}\text { Rp. } \\
497.361\end{array}$ \\
\hline 62 & Curcuma Soho Tab & Tablet & Rp. 1.528.592 & Rp. 2.140 .762 & $\begin{array}{l}\text { Rp. } \\
612.169\end{array}$ \\
\hline 63 & $\begin{array}{l}\text { Paracetamol If 500mg Tab } \\
\text { Str }\end{array}$ & Tablet & Rp. 1.489 .102 & Rp. 2.024.586 & $\begin{array}{l}\text { Rp. } \\
535.483\end{array}$ \\
\hline 64 & Cefadroxil Kap 500 Mg & capsule & Rp. 1.446 .460 & Rp. 1.955 .392 & $\begin{array}{l}\text { Rp. } \\
508, .932\end{array}$ \\
\hline 65 & Metformin $\mathrm{Tab} 500 \mathrm{Mg}$ & Tablet & Rp. $1.416, .562$ & Rp. 1.891.807 & $\begin{array}{l}\text { Rp. } \\
475.245\end{array}$ \\
\hline 66 & Piralen $10 \mathrm{Mg}$ Tab & Tablet & Rp. 1.401 .963 & Rp. 1.892 .050 & $\begin{array}{l}\text { Rp. } \\
490.087\end{array}$ \\
\hline 67 & $\begin{array}{l}\text { Harnal Ocas Tab } \\
\text { (Tamsulosin Hcl 0.4 Mg) }\end{array}$ & Tablet & Rp. 1.370 .007 & Rp. 2.050.522 & $\begin{array}{l}\text { Rp. } \\
680.515\end{array}$ \\
\hline 68 & $\begin{array}{l}\text { Domperidone Novell 10mg } \\
\text { Tab } \\
\text { Paracetamol Kimia } \\
\text { Farma500mg }\end{array}$ & capsule & Rp. 1.330 .833 & Rp. 1819.275 & $\begin{array}{l}\text { Rp. } \\
488.442 \\
\text { Rp. } \\
534.191\end{array}$ \\
\hline
\end{tabular}




\begin{tabular}{|c|c|c|c|c|c|}
\hline 70 & Ampicillin Kf 500mg Tab & Tablet & Rp. 1.249.444 & Rp. 1.737.693 & $\begin{array}{l}\mathrm{Rp} . \\
488.249\end{array}$ \\
\hline 71 & Dramasine Tab & Tablet & Rp. 1.188.381 & Rp. 1.702.454 & $\begin{array}{l}\text { Rp. } \\
514.073\end{array}$ \\
\hline 72 & Amlodipine If 10mg Tab & Tablet & Rp. 1.198.685 & Rp. 1.704.019 & $\begin{array}{l}\text { Rp. } \\
505.334\end{array}$ \\
\hline 73 & Allopurinol Tab $100 \mathrm{Mg}$ & Tablet & Rp. 1.153.042 & Rp. 1.639 .815 & $\begin{array}{l}\text { Rp. } \\
486.773\end{array}$ \\
\hline 74 & $\begin{array}{l}\text { Piracetam Novell } 800 \mathrm{Mg} \\
\text { Tab }\end{array}$ & Tablet & Rp. 1.088.598 & Rp. 1.594.151 & $\begin{array}{l}\text { Rp. } \\
505.553\end{array}$ \\
\hline 75 & $\begin{array}{l}\text { Bactoprim } 480 \mathrm{Mg} \text { Tab } 100 \\
\mathrm{~S}\end{array}$ & Tablet & Rp. 1.084.236 & Rp. 1.573.989 & $\begin{array}{l}\text { Rp. } \\
489.753\end{array}$ \\
\hline 76 & Depakote $\mathrm{Er} 250 \mathrm{Mg}$ & Tablet & Rp. 1.027 .448 & Rp. 1.647.297 & $\begin{array}{l}\text { Rp. } \\
619.849\end{array}$ \\
\hline 77 & Griseofulvin 500mg Tab & Tablet & Rp. 1.016.745 & Rp. 1.539.184 & $\begin{array}{l}\text { Rp. } \\
522.438\end{array}$ \\
\hline 78 & Ritez 10mg Tab 30s & capsule & Rp. 1.016.437 & Rp. 1.574.,993 & $\begin{array}{l}\text { Rp. } \\
558.555\end{array}$ \\
\hline 79 & $\begin{array}{l}\text { Isosorbid Dinitrat Tab 5mg } \\
\text { (Isdn) }\end{array}$ & Tablet & Rp. 1.022.134 & Rp. 1.511 .767 & Rp.489.633 \\
\hline 80 & $\begin{array}{l}\text { Cotrimoxazole Erita } 480 \\
\text { Mg Tab 100s }\end{array}$ & Tablet & Rp. 929.315 & Rp. 1.419.091 & $\begin{array}{l}\text { Rp. } \\
489.776\end{array}$ \\
\hline 81 & Ranitidin Tab $150 \mathrm{Mg}$ & Tablet & Rp. 894.152 & Rp. 1.386.267 & $\begin{array}{l}\text { Rp. } \\
492.114\end{array}$ \\
\hline 82 & $\begin{array}{l}\text { Ofloxacin } 200 \mathrm{Mg} \text { Tab } \\
\text { Novell }\end{array}$ & Tablet & Rp. 840.470 & Rp. 1.337.197 & $\begin{array}{l}\text { Rp. } \\
496.727\end{array}$ \\
\hline 83 & $\begin{array}{l}\text { Gabapentin } 300 \text { Mg Kapsul } \\
\text { (Ekat) }\end{array}$ & capsule & Rp. 804.402 & Rp. 1.311.714 & $\begin{array}{l}\text { Rp. } \\
507.312\end{array}$ \\
\hline 84 & Bisoprolol Tab 5mg & Tablet & Rp. 761.990 & Rp. 1.259.020 & $\begin{array}{l}\text { Rp. } \\
497.030\end{array}$ \\
\hline 85 & Pyrazinamid Tab $500 \mathrm{Mg}$ & Tablet & Rp. 739.627 & Rp. 1.257.330 & $\begin{array}{l}\text { Rp. } \\
517.703\end{array}$ \\
\hline 86 & Citicoline Tab $500 \mathrm{Mg}$ & Tablet & Rp. 661.900 & Rp. 1.280 .743 & $\begin{array}{l}\text { Rp. } \\
618.843\end{array}$ \\
\hline 87 & $\begin{array}{l}\text { Glibenclamideindofarma } \\
5 \mathrm{mg}\end{array}$ & Tablet & Rp. 661.683 & Rp. 1.156.566 & $\begin{array}{l}\text { Rp. } \\
494.883\end{array}$ \\
\hline 88 & Cetymin Tab & capsule & Rp. 618.889 & Rp. 1.174.176 & $\begin{array}{l}\text { Rp. } \\
555.288\end{array}$ \\
\hline 89 & $\begin{array}{l}\text { Captopril Errita 25mg Tab } \\
100 \mathrm{~s}\end{array}$ & Tablet & Rp. 520.960 & Rp. 1.031.328 & $\begin{array}{l}\text { Rp. } \\
510.368\end{array}$ \\
\hline 90 & Ibuprofen 200mg Tab & Tablet & Rp. 498.497 & Rp. 995.289 & $\begin{array}{l}\text { Rp. } \\
496.792\end{array}$ \\
\hline 91 & $\begin{array}{l}\text { Amlodipine Berno 10mg } \\
\text { Tab }\end{array}$ & Tablet & Rp. 442.950 & Rp. 973.682 & $\begin{array}{l}\text { Rp. } \\
530.731\end{array}$ \\
\hline 92 & $\begin{array}{l}\text { Allopurinol Berno 100mg } \\
\text { Tab }\end{array}$ & Tablet & Rp. 448.921 & Rp. 947.059 & $\begin{array}{l}\text { Rp. } \\
498.138\end{array}$ \\
\hline 93 & Acyclovir Tab $400 \mathrm{Mg}$ & Tablet & Rp. 421.313 & Rp. 936.703 & $\begin{array}{l}\text { Rp. } \\
515.390\end{array}$ \\
\hline 94 & Dexymox Forte 500mg & capsule & Rp. 407.646 & Rp. 925.699 & $\begin{array}{l}\text { Rp. } \\
518.053\end{array}$ \\
\hline 95 & Fenamin 500mg Tab 100s & Tablet & Rp. 383.559 & Rp. 895.793 & $\begin{array}{l}\text { Rp. } \\
512.234\end{array}$ \\
\hline 96 & Lerzin 10mg Cap 50s & Tablet & Rp. 381.593 & Rp. 884.125 & $\begin{array}{l}\text { Rp. } \\
502.532\end{array}$ \\
\hline
\end{tabular}




\begin{tabular}{|c|c|c|c|c|c|}
\hline 97 & $\begin{array}{l}\text { Vectrine Cap } 300 \mathrm{Mg} \\
\text { (Erdostein) }\end{array}$ & capsule & Rp. 366.512 & Rp. 937.507 & $\begin{array}{l}\text { Rp. } \\
570.995\end{array}$ \\
\hline 98 & Furosemid Tab $40 \mathrm{Mg}$ & Tablet & Rp. 347.368 & Rp. 857.549 & $\begin{array}{l}\text { Rp. } \\
510.181\end{array}$ \\
\hline 99 & Yusimox 500mg Tab 100s & Tablet & Rp. 321.376 & Rp. 838.656 & $\begin{array}{l}\text { Rp. } \\
517.280\end{array}$ \\
\hline 100 & Irbesartan $150 \mathrm{Mg}$ & Tablet & Rp. 315.345 & Rp. 846.743 & $\begin{array}{l}\text { Rp. } \\
531.398\end{array}$ \\
\hline 101 & Trisela $10 \mathrm{mg}$ & Tablet & Rp. 267.761 & Rp. 784.501 & $\begin{array}{l}\text { Rp. } \\
516.740\end{array}$ \\
\hline 102 & Cetrol Tab & Tablet & Rp. 247.564 & Rp. 768.608 & $\begin{array}{l}\text { Rp. } \\
521.044\end{array}$ \\
\hline 103 & Meloxicam Tab $15 \mathrm{Mg}$ & Tablet & Rp. 236.077 & Rp. 764358 & $\begin{array}{l}\text { Rp. } \\
528.281\end{array}$ \\
\hline 104 & Meloxicam Tab 7,5 Mg & Tablet & Rp. 153.643 & Rp. 665,985 & $\begin{array}{l}\text { Rp. } \\
512.342\end{array}$ \\
\hline 105 & $\begin{array}{l}\text { Mefenamic Acid Hexpharm } \\
\text { 500mg Capl 100s }\end{array}$ & capsule & Rp. 134.864 & Rp. 653,747 & $\begin{array}{l}\text { Rp. } \\
518.883\end{array}$ \\
\hline 106 & $\begin{array}{l}\text { Hydrochlorothiazide Tablet } \\
25 \mathrm{Mg}\end{array}$ & Tablet & Rp. 117.355 & Rp. 633.192 & $\begin{array}{l}\text { Rp. } \\
515.837\end{array}$ \\
\hline 107 & Antalgin Kf 500mg Tab & Tablet & Rp. 112.541 & Rp. 630.413 & $\begin{array}{l}\text { Rp. } \\
517.871\end{array}$ \\
\hline 108 & Amlodipine Dexa $5 \mathrm{Mg}$ & Tablet & Rp. 78.315 & Rp. 594.648 & $\begin{array}{l}\text { Rp. } \\
516.333\end{array}$ \\
\hline 109 & $\begin{array}{l}\text { Propanolol Dexa } 10 \mathrm{Mg} \\
\text { Tab }\end{array}$ & Tablet & Rp. 47.371 & Rp. 554.818 & $\begin{array}{l}\text { Rp. } \\
507.447\end{array}$ \\
\hline 110 & Salbutamol Tab $4 \mathrm{Mg}$ & Tablet & Rp. 25.117 & Rp. 534.661 & $\begin{array}{l}\text { Rp. } \\
509.544\end{array}$ \\
\hline & Total & & Rp. 546.086.226 & Rp. 603.633.813 & $\begin{array}{l}\text { Rp. } \\
57.547 .587\end{array}$ \\
\hline
\end{tabular}

\section{FINDINGS AND INTERPRETATION}

Based on the results of the calculations above, the results of the optimal order size, the number of products that will expire, the number of medicine returns, and the cost of supplies. With an optimal lot size, orders can be placed according to the demand. And on the other hand, it will reduce the risk of loss, damage, storage of medicinal products in sufficient quantities so that the products do not accumulate so that it creates investment in pharmacies. With the method currently applied by pharmacies, the total cost of supplies is Rp. 603,633,813, -. Meanwhile, based on the EOQ method, the total cost of inventory per year is Rp. 546,086,226, -. So that the method used in this study can save the total annual inventory cost of Rp. 57,547,587, - or about $9.6 \%$. The use of EOQ analysis can reduce expiration costs, can determine which medicines can be returned to suppliers based on existing terms and conditions, can guarantee the total cost of supplies.

\section{CONCLUSION}

The results of the $\mathrm{ABC}$ analysis show that there are 22 types of generic medicines belonging to group (20\%), 28 types of generic medicines belonging to group B (25\%) and 60 types of generic medicines belonging to group C (55\%). and based on the Economic Order Quantity (EOQ) method, the optimum order quantity for all generic medicines groups A, B and C varies from 13 - 2066 items per order. Reorder Point (ROP) or for all generic drug groups varies from 0-163 medicines. and for SS (Safety Stock) calculations, starting from 0 - 74 drugs. Meanwhile, for medicines that will expire at the end 
of the period for all generic medicine groups starting from 2 - 344 items. Then the drug that will expire can be returned to the supplier with a maximum of 10 items. and by calculating using EOQ can reduce the total cost of inventory by $9.6 \%$ or Rp. $57.547 .587,-$

\section{ACKNOWLEDGMENT}

This research received no specific grant from any funding agency in the public, commercial, or notfor-profit sectors.

\section{REFERENCES}

Alfanda, D., Pujotomo, D., \& Wp, S. N. (2018). Pengendalian Obat Dengan Menggunakan Economic Order Quantity ( EOQ ) Probabilitas Berdasarkan Analisis ABC Dengan Mempertimbangkan Masa Kadaluwarsa Dan Pengembalian Produk ( Studi Kasus : Instalasi Farmasi Rumah Sakit Nasional Diponegoro ). Jurnal Penelitian, 7(3).

Apriyani, N., \& Muhsin, A. (2017). Analisis Pengendalian Persediaan Bahan Baku Dengan Metode Economic Order Quantity Dan Kanban Pada PT Adyawanisa Satamping Industries. Opsi, $10(2), 128$.

Assauri, S. (2004). Manajemen Produksi dan Operasi (Revisi). Jakarta: Fakultas Ekonomi Univesitas Indonesia.

Buwono, R. I., Priyandari, Y., \& Jauhari, wakhid A. (2014). Usulan Perencanaan dan Pengendalian Persediaan Obat pada Gudang Farmasi Klinik XYZ dengan Menggunakan Metode EOQ. Teknik, 13(1), 29-39.

Dyatmika, S. B., \& Krisnadewara, P. D. (2017). Pengendalian Persediaan Obat Generik Dengan Metode Analisis ABC, Metode Economic Order Quantity (EOQ), dan Reorder Point (ROP) Di Apotek XYZ Tahun 2017. MODUS, 30(1), 87-95.

Heizer, J. . (2010). Manajemen Operasi. Jakarta: Salemba Empat.

Hermanto, Indrajaya, D., \& Suhendar, E. (2018). Analisa Pengendalian Manajemen Persediaan Dengan Menggunakan Metode Economic Order Quanty (Studi Kasus di PT. XYZ. Teknik, 7(1), $1-6$.

Nafisah, L., Puryani, \& Lukito, F. X. K. B. (2011). Model Persediaan Single-Item Dengan Mempertimbangkan Tingkat Kadaluwarsa Dan Pengembalian Produk. Prosiding SEMNAS Manajemen Teknologi.

Rachmawati, S. A., Syafirullah, L., \& Faiz, M. N. (2020). Perancangan Sistem Pengendalian Persediaan Barang Menggunakan Metode EOQ dan ROP Berbasis Web. Seminar Nasional Terapan Riset Inovatif (SENTRINOV), 6(1).

Rangkutty, F. (2017). Manajemen Persediaan: Aplikasi di Bidang Bisnis. Jakarta: PT Raja Grafindo Persada.

Resmana, D., \& Rukmayadi, D. (2019). Analisis Pengendalian Persediaan Obat generik Dengan Metode ABC Dan Metode Economic Order Quantity (EOQ) Di Apotek Anugerah Farma Bintaro. Teknik Industri, 228-235.

Sabarguna, B. S. (2004). Sistem bantu Keputusan Untuk Quality Assurance Pelayanan Rumah Sakit. Yogyakarta: Konsorsium Rumah Sakit Islam Jateng-DIY.

Sukanta. (2017). Pengendalian Persediaan Bahan Baku Menggunakan Metode Continuous Review System di Moga Toys Home Industry Teknik Industri. Fakultas Teknik. Universitas Singaperbangsa. Karawang.

Ulfa, M., Said Salim Dahda, \& Widyaningrum, D. (2018). Analisis Perecanaan Dan Pengendalian Obat Dengan Metode ABC (Always Better Control) Dan EOQ Probabilistik (Studi Kasus: Logistik Farmasi Rumah Sakit Muhammadiyah Gresik). Teknik.

Vrat, P. (2014). Basic Concepts in Inventory Management. Materials Management, hal. 21-36 